\title{
Deep Sea Water: Potential of Vietnam
}

Tran Ngoc Son

Dong A University, Da Nang, Vietnam

*Corresponding author: Tran Ngoc Son, Dong A University, Da Nang, Vietnam, Tel: 84913474023; E-mail: sontn@donga.edu.vn

Received date: Nov 15, 2017; Accepted date: Jan 15, 2018; Published date: Jan 22, 2018

Copyright: @ 2018 Son TN. This is an open-access article distributed under the terms of the Creative Commons Attribution License, which permits unrestricted use, distribution, and reproduction in any medium, provided the original author and source are credited.

\begin{abstract}
Exploitation of deep-sea water is a newly-developed industry in some countries such as USA, Korea and Japan. With the intention of serving the needs of medical treatment, pharmaceuticals, high end beverages, pure salt production, distilled water to preserve foods, etc. Meanwhile, in Vietnam, despite the potential of a country with over $3.444 \mathrm{~km}$ coastline and continental shelf areas with depths less than $200 \mathrm{~m}$ which accounts for more than half of the deep sea, deep-sea water industry is still unknown. Vietnam has a great potential to become a country of deep-sea water mining in its marine economic strategy; to implement the goals of Resolution No. 09-NQ/T.U dated on 09-02-2007 Central Executive Committee about "Vietnam marine Strategy until 2020" with the goal of 2020, Vietnam will become a strong nation, enriched from marine.
\end{abstract}

Keywords: Deep sea water; Deep-sea water industry; Country of deep-sea water mining; Marine economic strategy

\section{Introduction}

\section{The deep sea water}

Ocean (according to documents from open encyclopedia) is divided into several areas or floors, depending on the physical conditions and biological properties of these areas. Seas include all areas of sea water (excluding the seabed) and it can be further divided into the next area from top to bottom with depth and illumination sequence as follows.

Zone lighting (epipelagic) called sea covered ocean surface from the sea surface to a depth of $200 \mathrm{~m}$. This is the area in which photosynthesis takes place and therefore contains the greatest biodiversity in the ocean. Because plants only survive via the photosynthesis process, any life entity which was found below this depth lives on sinking substances from the top or finding the other sources. An underexposed area at depths below $200 \mathrm{~m}$ which can't be reached by sunlight is the area closed to exposed areas.

Twilight zone(mesopelagic) is on the top adjacent to the exposed areas, with the lowest in the boundary layer temperature is $12^{\circ} \mathrm{C}$, in which tropical areas is generally located at a depth of between 700 and 1,000 metres. This is the region's aquifers underexposed special structure than the surface water layer in the ocean does not happen by photosynthesis in the water layer. This water layer is called the deep sea [1].

Midnight zone (bathypelagic) lies between $10^{\circ} \mathrm{C}$ and $4^{\circ} \mathrm{C}$, or between 700-1000 metres depth of 2,000-4,000 metres.

Abyssal zone (abyssal pelagic) with its lower boundary lies at a depth of about 6,000 metres.

Hadal zone (hadal pelagic), also known as the ocean trenches. It is located between a depths of 6,000 metres and 10,000 metres and is the deepest part of the ocean.

Based on the division of the ocean floors as above, we can define the deep sea water (DSW) is located in waters off central coast (mesopelagic) at depths ranging from $200 \mathrm{~m}$ to $700 \mathrm{~m}$ where plants is difficult to survive by photosynthesis which is unlikely at this stage.

\section{Materials and Methods}

By analysing and comparing primary data and forecasting methods for economic trends for deep seawater in the application of this new product in the world.

\section{Results}

\section{History detection DSW world}

The discovery of deep sea water is a very fortuitous event in the scientific field of mining. The US is the first country to conduct deepsea mining, originated from a science project in progress of local governments that do not have to start from DSW. In 1974, the United States was boycotted oil activity by OPEC. Due to the lack of fuel to produce electricity, Hawaii state government established a laboratory in the hope that it will find alternative energy to produce electricity.

We already know that the basis for experiments is based on a theory invented by Jacques-Arsene 1880 d'Arsonval, a French scientist, which produce electricity by hot water and cold water. Hawaii Scientists used hot water in Hawaii and $100^{\circ} \mathrm{C}$ cold water taken from the seabed to carry out the experiment. But experimental project to generate energy from the sea eventually fail. However, the pipe length of 600 meters to take deep water in the sea water is still there.

Initially, Hawaii biologists and aquaculture used the pipe to put water in fish, clams and seaweed areas and they have discovered that deep sea water supplies a very good nutrient than normal water. Since then, it has awakened the curiosity of scientists and American scientists began to study [2].

\section{The function of DSW}

Through the research of scientists regarding marine resources mining, the deep sea water ion balances in depth of more than 200 meters below the ocean where sunlight is not coming down. At the 
same time, deep sea water has the same texture as the fluid in the skin, with purity; high mineral content and high absorption into the skin than ordinary water. So deep sea water helps solving the problem of dry skin due to rapid moisture mechanism, maintains ion balance (mineral) similar to the body. The study also points out, deep sea water increases the moisture content of $17 \%$ compared with normal water.

Preparations with a basis of deep sea water can bring practical benefits in protecting human health which experts use as a solution in the treatment of post-operative support activity, sinusitis disease and prevention of respiratory diseases and other related respiratory diseases based on minerals and trace elements in seawater [3]

Deep sea water is exploited at reasonable depth below 200 metres calculated from the sea. After that, it is sterilized by UV (Ultra Violet radiation)-UV is emitted by the sun, divided into 3 categories: Ultra Violet A (UVA), Ultra Violet B (UVB) and Ultra Violet C (UVC). Though UVC rays are the most dangerous ultraviolet to humans, there is no potential harm to human because it was blocked by ozone layer. However, UVA and UVB rays can also cause cancer. Then, deep sea water is treated by ozone and filtered through ultrafiltration device with a diameter of 0.1 micrometres. In particular, the process in a modern scientific and helped preserve almost all trace elements as $\mathrm{Zn} 2+$ (zinc) and $\mathrm{Cu} 2+$ (Copper). Also thanks to the participation in a "cornucopia" of the trace elements, deep sea water obtains disinfection effects, anti-inflammatory, astringent mucosa, mucosal recovery, thus can prevent runny nose, stuffy nose and sinus and the disease spread via res respiratory system.

\section{Extraction and application of DSW in countries around the world}

In 2001, Yasuki Takano-employer of Koyo USA corporationaccidentally discovered pipes and acquired the right to use it to produce sea water bottled by special technology since 2003 .

After the USA, there are many potential companies exploit the deep sea water and sea products from Japan and Korea. Gangwon-Deep Sea Water Company of Korea was established in 2006 as one of the pioneering firms in this new field to spend about \$25 million investment to exploit deep sea water. The company not only produces bottled water but also launched into the market other deep-sea beverages such as high-end beer, wine, food production, used as cosmetics, pharmaceuticals, pure salt. Chairman of deep Sea water Gangwon company explained: due to be exploited at a depth of 600 meters, with a temperature below $2^{\circ} \mathrm{C}$, water of the Pacific Ocean contains many minerals such as phosphorus and calcium and should be very good for human health. Moreover, it is especially pure [4].

In recent years, Korean Gangwon Company, by threaded a nozzle with a special funnel depths of over 600 meters in about $6 \mathrm{~km}$ offshore, deep sea water is drawn ashore about 3,000 tons per day through a special pump system. A factory on the banks of duty extract raw water into three product groups: group was pure deep sea water desalinization to drink, the deep sea water for food processingcosmetic-medicine and raw water group for bathing-fish salting cosmetics making.

Develop desalinization deep sea water products for drinking purpose: Many countries prioritize this field because technology for processing is not only too complicated but also create products for everyone. The price of a branded "Artic Spring" bottle in Korea now cost about 20 thousand VND, about three to four times higher than the price of purified water bottle in Vietnam. However, Korean still prefer, especially mothers during lactation period, athletes, actors and businessmen. Because deep sea water contains mineral content higher than 20 to 30 times normal drinks. In addition, magnesium is abundant in deep sea water also makes the skin fresh and bright [5].

Develop food processing-cosmetic-medicine deep sea water products: These product groups are interested as drinking groups. With stuffy nose case due to increased secretion, deep sea water can dilute and excrete fluids out. When dry weather or regularly working in airconditioned rooms make the dry nasal mucosa irritation, discomfortthis time deep sea water will work to help restoring moisture nose. Moreover, menthol, essential oils of pig-shit flower which are added to water to steam deep sea aerosol will help creating a sense of cool for the sick and good intervention flu symptoms are common for children people. Because deep sea water nasal hygiene solution is very rich in minerals and contains over 60 trace elements, vary with the depth and distance. In particular, it contains copper and zinc elements, which acts as astringent mucosa, mucosal recovery, good antiseptic and antiinflammatory. Many clinical studies showed that deep sea water in the prevention and effective treatment support the symptoms of sinusitis, and reduce the rate of relapse. Deep sea water has long been the trusted professionals as a solution to support treatment and postoperative respiratory diseases based on the antiseptic, antiinflammatory of the trace elements in seawater composition [6].

Develop bathing-cosmetics fish salting deep sea water products: Other applications of deep sea research from powdered milk cleanser and charcoal face wash. Japanese use it for years. The leading cosmetics companies in Japan used deep sea water in skin care products to replace their traditional products.

We all know that deep sea water not only contains iodine, but also all kinds of minerals extracted from the earth's crust, such as iron, copper, magnesium, sodium, even the silver, gold and radium. However, whatever overuse can cause adverse reactions.

Deep sea water has improved features from the nature of the skin to the outside. Its effect is slow but deep and durable when used for bathing. It prevents and corrects mild to moderate skin, bring positive results in fixing your face into an oval when unfortunately you face was saggy because of obesity.

Deep sea water is also effective in toning muscles on women's breasts, hard tone cuticle. In summer, when you bath in the deep sea water on vacation, you can change the little in the top half of the body. Deep sea water is comparable as hormones, making the body more active. Deep sea water makes the hair shiny. Some beauty salons also use deep sea as the background for the treatment of chronic inflammation skin disorder [7].

\section{Status of deep sea water industry in VN}

The research relevant to DSW industry in Vietnam: According to scientists, due to water depths of at least $200 \mathrm{~m}$ below the surface and no sunlight and stable temperature below $2^{\circ} \mathrm{C}$ throughout the year, deep seawater (DSW) is pure water and almost sterile. When ocean currents move from the Greenland coast to the North Pacific Ocean, they create ultra-cold and coherent water flow. The kind of water flows under ocean floor forming deep sea water and not be mixed with surface water. This process lasted for thousands of years and existed until now. 
Page 3 of 4

In Vietnam (the study is based on data from before 1995) in stages over the past 10 years, sea level oscillations in Vietnam is more volatile due to the seabed topography is quite diverse. Greatest depth is 5560 $\mathrm{m}$, the continental shelf to a depth of $200 \mathrm{~m}$ below sea area accounted for more than half. In the northern Gulf of Thailand and the sea depth varies from a few meters to less than $100 \mathrm{~m}$.

There exists asymmetry in the distribution of the depth of the sea between the west and east; between the north and south of the sea. Sea is in the east and southeast have slopes greater than the bottom of the sea in the west and northwest. This feature greatly affects oscillation tide mode and sea level of South China Sea.

The temperature measurements show that the seabed temperature depends on the depth in the South China Sea, (the seabed temperature changes from 10.5 to 7.5 degrees Celsius from $300-500 \mathrm{~m}$ and from 5 to 2.5 degrees Celsius from 1.000-3.000 m). In the deeper part of the valley in the South China Sea, the temperature drops below 2do C. continental slope area, the change in temperature high value, from 60 to 94 degrees $\mathrm{C} / \mathrm{km}$. So over $300 \mathrm{~m}$ deep water part of the South China Sea Vietnam meets the conditions of temperature for gas hydrate formation [8].

According to scientists, most of the seafloor topography on the waters of Vietnam parallel rift coincides with the direction of the South China Sea. There appear more volcanic terrain types which is favourable for the formation of underground plateau, raising zones. Part continental slope Central and Southeast, seabed topography changes abruptly from a few hundred meters down 1500-2.500 m, forming steep cliffs. This area young volcanic formation, this terrain is favourable for the formation of sedimentary conical structure. The structure is very suitable for the formation of gas hydrate in the highlands of groundwater, raising zones, cones seabed sediments, volcanic mud. In particular, the southern part of the Spratly islands exist structures "wedge growth" is one of the topographic structure is very favourable for the existence of gas hydrate.

Gas hydrate is a crystalline substance including water and methane molecules are usually found beneath the permafrost and geological layers deep in the ocean, a source of alternative materials for oil and coal. Gas hydrate is the world's scientists as one of nine clean energy futures [9].

Based on the scientific study of the depth of the continental shelf in Vietnam, we see that it is possible to think about forming deep-sea exploitation industry. Clearly, with more than half of Vietnam's sea area with a depth of $200 \mathrm{~m}$ from the continental shelf down to $5.560 \mathrm{~m}$ is a "Golden State" of deep sea water.

In addition, the study of the science we see that besides exploiting deep sea we have a result that is more gas hydrate-a clean energy for future in the same depth exploitation. It looks like "kill two birds with one stone."

The market for products from DSW in Vietnam: Currently, the product of deep sea water only appears through the use of products in the pharmaceutical industry. The most common are deep sea water products curing respiratory common diseases.

In Vietnam, there were some pharmaceutical products with ingredients from the deep sea, which deep sea water nasal spray is a typical one. Type of sea water nasal hygiene is the best type of water extracted from the deep sea in the form of undiluted (100\% seawater). This type of content and trace minerals such as copper, manganese, magnesium, sulphur, selenium... more cleaning effect than diluted sea water in antiseptic, anti-allergic, anti-inflammatory, moisturizing the mucosa.

In particular, Xisat deep sea water product is certificated for circulation by the Ministry of Health in Vietnam. According to the Nielsen survey (Omnibus 3/2012), Xisat deep sea water product currently accounts for $80 \%$ market share in Hanoi and $96 \%$ market share in Ho Chi Minh City in total product solution nasal hygiene. Over $90 \%$ of consumers recognize the use of Xisat deep sea water product daily to prevent runny nose, nasal congestion due to weather changes or dust pollution. Since then, we see that in the two largest cities in Vietnam, deep sea water ratio y-pharmaceutical industry has a very high market share in the group of nasal hygiene product that is a very good sign for the market consumer majority in $\mathrm{VN}$ compatible with the respiratory hygiene and thereby help guide to develop deep sea water industry.

Also, in the opinion of the experts, the sea itself contains a lot of minerals. However, if exploited at a reasonable depth (about $450 \mathrm{~m}$ above the sea), the sea now known as deep sea water will become the most benefits for Vietnam at this depth in the composition of seawater more 60 trace elements such as $\mathrm{Cu}, \mathrm{Zn}, \mathrm{Al}, \mathrm{Ag}, \mathrm{Mn}, \mathrm{Pb}$. Meanwhile, the medical community has also made recommendations: All of this material proved extremely effective in the treatment and prevention diseases related to postoperative respiratory and sinus area [10].

It can be said that the deep sea water is very useful for the development of the pharmaceutical industry.

\section{The solution for DSW industry in Vietnam}

Exploiting the advantages of coastal economic zone in Vietnam: Currently, in Vietnam up to May 2017, there are 18 economic zone mostly along the coast throughout the length of the country from Quang Ninh to Ca Mau and even Phu Quoc has one. This means that in Vietnam where there is the sea there is economic zone. Once again, it shows a great ability to develop DSW industry in the heart of the coastal economic zones in VN. In addition, other cities which don't have economic zone such as $\mathrm{Da}$ Nang can develop this industry to become the economic centre of Vietnam.

It is clear that the development of mining deep sea water industry would have other plants evolved from an aqueous extract as pharmaceuticals, cosmetics industry, food processing, mineral water treatment industry, premium beer, wine, pure salt, such a series of other plants grow into an "effect" that we do not need to call a sector investment into the economy.

18 coastal economic zone in VN include: Chu Lai (Quang Nam), Dung Quat (Quang Ngai), Nhon Hoi (BinhDinh), Chan May-Lang Co (Thua Thien-Hue), Phu Quoc-Nam An Thoi (Kien Giang), Vung Ang (Ha Tinh province), Van Phong (Khanh Hoa), Nghi Son (Thanh Hoa), Van Don (QuangNinh), Dong Nam Nghe An (Nghe An), Dinh Vu-Cat Hai (Hai Phong), Nam Phu Yen (Phu Yen), Hon La (Quang Binh), Dinh An (TraVinh), Nam Can (Ca Mau), Dong Nam (Quang Tri), Thai Binh (Thai Binh), Ninh Co (Nam Dinh) need a new research and industry will be the future owners of the plant in the deep sea mining strategic development of its economy.

Recently, Xisat deep sea water product of Merap Group has become the first hygiene solution produced in Vietnam certificated by Ministry of Health. Xisatis high appreciated because it is the first product exploited in Thai Binh, from sea level of 450 metres. This is a good sign for the economic zone in the Pacific Coast industry's deep sea where 
Page 4 of 4

you can create contagious to other coastal economic zone in $\mathrm{VN}$ in deepwater industry.

Industrial development in the field of service life: However, in practice we find that the deep sea products are only known in the pharmaceutical field VN in treating common diseases. Meanwhile, the bottled water industry in $\mathrm{VN}$ annually is a very urgent problem in the "confrontation" with the uncontrollable quality products

Vietnam remains a potential consumer market because a person drank 20 liters of non-alcoholic bottled beverage /year, while the average consumption per person in the country on the world is over 40 liters /year market so bottled beverage market is still full of potential. Moreover, Vietnam standard on bottled water to drink soft drinks for people on a much lower quality than the standard normal water in Europe alone not to mention the bottled water in life. We try to refer to the index of iron and manganese in a liter of water in Europe is very low (good) while drinking in $\mathrm{VN}$ two indicators are very high (not good).

(EU: iron $0.3 \mathrm{mg} /$ liter -TCVN: iron $0.5 \mathrm{mg} / \mathrm{liter}$ )

(EU: manganese $0.1 \mathrm{mg} /$ liter -TCVN: iron $0.5 \mathrm{mg} / \mathrm{liter}$ ).

While iron and manganese $\mathrm{n}$ the deep sea water exists naturally and necessarily in the beverage without the artificially synthesized by the technology unnecessarily expensive and not quality by DSW. In addition, the cosmetics industry, food processing industry, medical industry mineral water, beer, pure salt, is a huge gap in the economic strategy of Vietnam. VN cosmetics market is currently being evaluated with a lot of potential, great demand with sales estimated average period 2009-2011 is nearly 130-150 million/year, the rate of sales increased by $30 \%$. Accordingly, $\mathrm{VN}$ had several advantages to developers, but this time more than $90 \%$ market share in the hands of the major brands in the world. When will Viet Nam has new cosmetics industry? That is the question should be the answer for the development of DSW in VN now is the right time and opportunity.

\section{Discussion}

It can be confirmed that the deep sea water industry is a new industry and will bring greater efficiency in current strategic maritime economy of many countries in the world. In addition to the United States, Japan and Korea are the two countries has been a pioneer in the field of the industry in Asia. With regard to Viet Nam, we shouldn't sit on the side lines and should be a pioneer in ASEAN to research and development industry development prospects. With the potential of sea, Vietnam has the right to expect in the near future to join the national deep sea mining pioneer in the world.

\section{References}

1. http://vi.wikipedia.org

2. Exploitation of deep sea water, new potential industry.

3. Deep-Sea prevention of respiratory transmitted diseases. Merap Group.

4. Truong Dinh Hien, Towards a sea development economy country.

5. Truong Dinh Hien, Towards a sea development economy country. Saigon Entrepreneur weekend.

6. Nguyen Thanh Son, Development sea mining industry.

7. Potential and practical condion of sea economy in Vietnam, cucktbvnlts.gov.vn.

8. KAILUA-KONA (2004) Hawaii Japan thirsty for Hawaiian deep-sea water, US Water News Online.

9. Deep ocean water From Wikipedia, the free encyclopedia.

10. Understanding deep sea water and the its future in the worldwide marketplace. 